\title{
How to attract them and keep them: the pharmacy attributes that matter to Australian residents with chronic conditions.
}

\section{ABSTRACT}

\section{Objective}

To explore the attributes of pharmacy choice for people with chronic conditions.

\section{Methods}

Semi-structured interviews were conducted between May-October 2012, across four regions in three Australian states. Purposive sampling was used to recruit participants with chronic conditions and unpaid carers. Interviews were analysed via the constant comparison method.

\section{Key Findings}

Ninety-seven interviews were conducted. The majority of participants were regular patrons of one pharmacy and five attributes influenced this choice: patient-centred care, convenience, price, personal trait or preference and service/medication need. Patient-centred care, such as providing individualised medication counselling, continuity of care, development of relationships and respectful advice, emerged as an important attribute. There was minimal discussion as to choosing a pharmacy based on the provision of professional services, underscoring the limited consumer knowledge of such services and related standards of care.

\section{Conclusion}

Patient-centred care is an important attribute of quality care as perceived by people who are regular community pharmacy users. These findings highlight the need for pharmacy staff to implement a patient-centred approach to care, thus meeting the perceived needs of their 
customers. A greater effort is also necessary to raise the profile of pharmacy as a healthcare destination.

\section{Keywords}

Patient-centred care, Pharmacists, Community pharmacy, Chronic disease 


\section{INTRODUCTION}

There is a global focus on implementing and evaluating healthcare services to better tackle health problems, such as chronic conditions, whilst meeting people’s needs and ensuring a quality healthcare experience. ${ }^{[1]}$ This is best demonstrated by the increasing emphasis towards delivering a patient-centred approach to care, currently perceived as an integral dimension of high quality healthcare worldwide. ${ }^{[2-4]}$

The pharmacy profession has increased its focus on patient-centred services to address healthcare needs of people with chronic conditions. ${ }^{[5]}$ This is particularly important given their increasing prevalence. ${ }^{[6]}$ Community pharmacy is ideally situated to assist people with managing the medications commonly used in the treatment or management of chronic conditions, ${ }^{[7]}$ such as asthma and diabetes. In order to support this, the Australian government has introduced remuneration for community pharmacy to provide a range of initiatives, ${ }^{[8]}$ such as disease management services and medication use reviews. ${ }^{[9]}$ These services enable community pharmacy to focus on prevention or management of chronic conditions or both, thus working with people to improve their health.

Despite recent changes, there is limited research on the quality of Australian pharmacy services $^{[10]}$ from a patient-centred perspective, even though patient-centred care can promote beneficial outcomes, such as improved satisfaction and quality of care. ${ }^{[1]}$ Historically, evaluations of community pharmacy and its services have focused on patient satisfaction. ${ }^{[12-}$

${ }^{16]}$ Yet, there are methodological concerns about using this outcome as an indicator of service quality, such as the lack of well-designed and validated satisfaction instruments. ${ }^{[17,18]}$ Other studies have investigated consumer perspectives of a specific professional service, without reference to the entire pharmacy experience. ${ }^{[19-21]}$ Whilst these studies provide significant contributions, it is also important to explore the experiences of community pharmacy as a whole, from the perspectives of people living with chronic conditions and their carers. An 
exploration of the consumer's experiences (patient-centred perspective) is essential to ensure and maintain a high quality healthcare service.

One approach to exploring pharmacy experiences is to focus on assessing a person's choice of pharmacy, ${ }^{[22-24]}$ which can be viewed as a quality indicator. Arneson et al discussed that a person remains loyal to a pharmacy if their needs are being met and they are satisfied. ${ }^{[22]}$ Both price ${ }^{[12,22]}$ and convenience ${ }^{[22-24]}$ are acknowledged as factors influencing pharmacy choice, with people having a strong preference for a pharmacy located near their home. ${ }^{[25]}$ However, considering the evolution of Australian pharmacy practice, most of this literature is dated. Furthermore, these studies do not consider how the attributes of patient-centred care influence pharmacy choice.

Given the current health climate and the evolving role of community pharmacy, it is more important to focus on pharmacy choice of people with chronic conditions and their carers rather than people needing assistance for minor ailments. This is because the latter are less likely to require continuing medication or pharmacy services. This study will provide a framework for the pharmacy profession to identify the quality features of patient-centred care or services that these consumers' value. This will enable pharmacy staff to build on these positive experiences and assist in understanding, and preventing, any recurrent negative consumer experiences. Consequently, this study aims to explore the attributes of pharmacy choice from a large qualitative study of people with a variety of chronic conditions and their carers.

\section{METHOD}

\section{Study participants}

Participants were purposively sampled ${ }^{[26]}$ from four Australian regions that represent diversity in geographical location, culture and socioeconomic status: Logan-Beaudesert and 
Mount Isa (Queensland), Northern Rivers (New South Wales) and greater Perth (Western Australia). Participant recruitment was achieved using project information mail-outs via contacting peak consumer health and carer organisations in the four project areas, and word of mouth from participants, i.e. snowball sampling. ${ }^{[27]}$ Community pharmacies were asked to distribute project information, and were contacted either by direct canvassing or via key pharmacy organisations. Eligibility criteria were established to promote diversity in the consumer sample and represent the Australian population that might be living with a chronic condition. ${ }^{[26]}$ This ensured a range of people with varying health conditions, age, and cultural background. Participants had to have one or more chronic condition(s), or be an unpaid carer, or both.

\section{Procedure}

An interview guide was informed by previous stakeholder research on the same topic (Panel

1), ${ }^{[28,29]}$ and data were elicited using semi-structured, in-depth interviews. To ensure that the interview guide was culturally appropriate, ${ }^{[30]}$ comments were sought from a Reference Group comprised of culturally diverse stakeholders with healthcare expertise. To promote a standardised interviewing method, the first 10 interviews were conducted by two researchers with experience in qualitative data collection (SM, AS), who then discussed their approach with the other interviewers (FK, CC, CM). The first 20 interviews were transcribed by the researchers to enable familiarisation with the initial data and further refinement of the interview guide. Debriefs were conducted with the research team (via email) after each interview, and feedback was obtained throughout the data collection process.

Individual interviews were conducted during May-October 2012, either via telephone or faceto-face. The interviewer and interview location were informed by the specific needs of the participant and consideration of their personal circumstances, such as cultural background or religious beliefs. Interviews were audio-recorded and ranged between 15-91 minutes (average 
50 minutes). Ethical approval was obtained from a University Ethics Committee (PHM/12/11/HREC).

[Insert Panel 1 here]

\section{Data analysis}

Interviews were transcribed verbatim either by professional transcribers or by the researchers (except for two participants who did not want the interview recorded; field notes were taken instead). A random sample of transcripts was checked for accuracy and a constant comparison method was used to analyse the data. ${ }^{[31,32]}$ This method involves returning to the transcripts frequently during the analysis process to check that the data substantiates the insights and inferences. ${ }^{[33]}$ The data were first openly coded into themes using the qualitative data analysis package QSR NVIVO $9^{\odot}$. To assist data familiarisation, researchers involved in coding did not code their own interviews. After initial coding, further interviews were explored to develop sub-themes, leading to further category refinement. A research team member (MK) checked the coding for randomly selected transcripts to ensure data credibility. The data analysis process was frequently discussed and reviewed between those researchers coding and also with the research team. Recruitment continued until data saturation was established, e.g. when participants' experiences and perspectives became recurring and were in line with previously collected data. ${ }^{[34]}$ To guide the analysis, two recent studies that categorised the attributes of patient-centred care differently were used: (i) a concept analysis by Morgan and Yoder, ${ }^{[35]}$ which identified four broad ranging attributes of patient-centred care, e.g. individualised, empowered, holistic and respectful care; and (ii) a qualitative study by Dancet et al., that defined ten attributes as either humanistic, e.g. emotional support and relationships, or organisational factors, e.g. accessibility and continuity of care. ${ }^{[36]}$ Importantly, the attributes identified by Dancet et al., ${ }^{[36]}$ could further describe Morgan and 
Yoder's ${ }^{[35]}$ framework or, in the case of most of the organisational factors, facilitate its development.

\section{RESULTS}

\section{Participants}

Ninety-seven interviews were undertaken (Table 1); the majority $(n=65)$ with female participants. Ages ranged from 16-85 years (mean=57.2 years; $\mathrm{SD}=13.03$ ). There was a diverse range of chronic conditions among participants, including: diabetes, mental illness, cardiovascular, respiratory and musculoskeletal conditions. Notably, almost $90.0 \%$ of participants had been diagnosed with two or more chronic conditions.

\section{[Insert Table 1 here]}

Participants could be classified according to one of three patron categories: those who were a regular patron of one pharmacy, those with a regular pharmacy who utilised other pharmacies for specific needs, or a casual visitor to various pharmacies. A number of factors determined this choice: patient-centred care, convenience, personal trait or preference, price and service/medication need.

\section{Patient-centred care}

A patient-centred approach was discussed widely amongst participants who patronised a regular pharmacy. A variety of attributes of patient-centred care from both Moran and Yoder $^{[35]}$ and Dancet et $a l^{[36]}$ were identified, which is exemplified in the following quote:

...she [pharmacist] makes a point to come over to me...she smiles, she's just nice, she's an educated pharmacist, she takes time to tell you when you're changing your drugs...she explained possible side effects... [C_1150] 
The above participant described both respectful and individualised care as defined by Morgan and Yoder. ${ }^{[35]}$ The encounter was respectful in terms of the pharmacist smiling and being nice, i.e. humanistic attributes, and being accessible for counselling purposes, i.e. organisational attributes. ${ }^{[36]}$ Furthermore, the participant appreciated being given medication advice, an organisational attribute that is a component of individualised care.

\section{Individualised care}

Taking the time to ensure that the person's individual needs were met and not identifying people solely by their condition(s) were exemplars of caring pharmacy staff. This was highly valued by participants, as this increased approachability and fostered the development of a relationship, attributes identified by Dancet et al. ${ }^{[36]}$

The provision of information ${ }^{[36]}$ was viewed positively by participants and deemed essential when obtaining a new medication:

...if I go up there with a script, she'll [pharmacist] come out...to explain what it's all for... [C_1053]

Continuity of care ${ }^{[36]}$ was another reason for utilising a regular pharmacy, as this facilitated awareness of the person's medical history.

...when you're away from your pharmacy and you go to a new pharmacy, they don't seem to have that amount of personal care... [CC_016]

This enabled staff to apply this knowledge to the unique needs of the individual, e.g. providing tailored medication information.

For others, medication safety was a key priority for them and hence, using a regular pharmacy was seen as a way to optimise this via continuity of care: 
One chemist, all your pills are together. The chemist can know what you're on; look for drug interactions... [C_1072]

Alternatively, other participants viewed this primarily as a matter of convenience, as it could minimise the burden of answering questions or explaining personal circumstances.

\section{Empowering care}

Providing empowering care in terms of support and encouragement was also important:

...because of being on the [opioid]...he always used to say to me, "(name) you're no junkie, you're the only one who reduces, everybody else they go up." And I knew that he knew that I would one day be normal again... [CC_1065]

Staff approachability facilitated a relaxed environment for consumers to ask questions and seek advice, thus supporting patient empowerment and resulting in continued use of that pharmacy:

...he [pharmacist] is very approachable and all the staff are too...you never feel as if you are stupid or asking a silly question...[C_005]

\section{Holistic care}

A holistic approach was being concerned about the person's overall wellbeing:

She's [pharmacist] a very caring person... who has an interest in the whole picture and bothers to keep that in her memory bank and not just on the computer. [C_015]

Furthermore, providing holistic care, where the pharmacy staff saw beyond the person's medical history, e.g. a disability, respected their role as a carer and as a person knowledgeable in their condition(s), fostered a continuing relationship. 


\section{Respectful care}

When pharmacy staff acknowledged the associated difficulties in being a 'perfect patient', this enhanced perceived staff approachability and respectful care:

...they [the pharmacist] were accepting of who you were and you are not an angel and you had done things wrong and that was okay you are human... [C_007]

One consumer with a disability articulated many reasons why she continued to go to a regular pharmacy; it was convenient, she was not judged, and the staff respected her knowledge of her own condition(s). Furthermore, she described the stigma and disrespect experienced elsewhere:

...there's still a lot of pharmacists, and I'm talking mainly about the young ones...think that therefore you must have an intellectual problem. Because you've got the physical disability and you can't move... [CC_1166]

The development of a relationship was particularly essential for participants of Aboriginal and Torres Strait Islander background. A community elder expressed the need for his/her people to be able to trust healthcare professionals before engaging in conversation about health matters:

...if they go there on a regular basis to that one pharmacy I think that's important...'cause Aboriginal people they need to trust people. And once they get the feeling they can trust people then they're more likely to open up. [C(IND)_2001]

The significance of a humanistic approach was also mentioned by two Aboriginal and Torres Strait Islander participants. They described that the simple act of a health professional placing 
a hand on their shoulder provided a sense of care and safety, and that this was an important attribute for optimal healthcare.

The preference for good, respectful customer service over convenience was highlighted by a participant who stopped patronising the pharmacy located next to her doctor's surgery, because of the abrupt and arrogant manner of the pharmacist:

...Even if I've got to come out [of] the doctors and hop on the bus to go to [location name]...I will rather than go there and put up with that [behaviour]. [C(IND)_1097]

\section{Convenience}

The majority of participants selected a conveniently located pharmacy, e.g. close to their home or doctor, to regularly use, in order to reduce the time accessing care. Remarkably, some participants described a lack of individualised care with their regular pharmacy, yet continued to utilise it because it would be a greater burden and inconvenience to change pharmacies:

...when we got there they haven't got it [medication]...It's annoying and frustrating...We haven't got another one [pharmacy] though. We go shopping at [location name] and we just stop over [at] the chemist, leave a script there... [C(CALD)_1085]

Participants without a regular pharmacy placed a higher value on convenience than on individualised service, or used pharmacies infrequently and accessed whatever one was open at the time:

...I don't go to the same one each time, cause if I have to go into one town for something.... I'll go to the pharmacy while I'm there. [CC_1034] 


\section{Price}

For some participants, particularly for low income earners, price was an important factor influencing pharmacy choice. Participants were frustrated when they were charged various prices for the same medication at different pharmacies. As a result, they were more inclined to shop around for the best price and stay at that particular pharmacy.

Trade-offs between pharmacies were also evident with respect to price and meeting different needs, and for some consumers, this resulted in feelings of disloyalty:

We pretty much go to one pharmacy which is up the road here.... The problem is it's a small pharmacy and so therefore all their vitamins...are a lot dearer...so we go to the other pharmacy which is I suppose rude on our behalf. But we're pensioners. [C_1053]

\section{Personal trait or preference}

The choice of pharmacy was influenced to a lesser extent by the personal trait or preference of the individual. Some consumers from culturally diverse backgrounds sought a pharmacy where a staff member spoke the same language or had the same cultural background. When asked about the possible impact of having a pharmacist of the same cultural background, one Samoan participant explained:

...it would draw all the Samoans in...then they'll ask questions and that's the good thing about it... [CC(CALD)_1124]

\section{Service/medication need}


Two or more pharmacies could be utilised by participants for a number of reasons: convenience, better medication price, or availability of different services such as medication compounding:

...The one we get the [compounded product] from is really weird...we spend a fortune...but...there's no interaction...they wouldn't know who I am if I walked in there...I still go to the pharmacy near our old house...they just know me... [CA_1013]

For some participants, an attribute of pharmacy choice was whether the staff met their service expectations; it was important to obtain exactly what they wanted, when they needed it:

...you just tell them what you need and they'll give it to you, or if you're not really a $100.0 \%$ sure on what it is, they talk through whatever's available...[C_1107]

\section{DISCUSSION}

This study provides a greater understanding of patronage choice for people with chronic conditions and carers. Participants could be categorised into three patron groups: a regular patron of one pharmacy, a casual visitor to various pharmacies, or both, i.e. they have a regular pharmacy but utilised other pharmacies to meet specific needs, such as better medication pricing or service provision. Our findings corroborate existing research indicating that people with complex conditions can place a higher importance on interpersonal skills when choosing a preferred healthcare provider, ${ }^{[37]}$ and value specific humanistic aspects of patient-centred care, such as smiling and nice staff. ${ }^{[36]}$ Furthermore, this study has identified that patient-centred care can influence choice of pharmacy, which was to date a relatively unexplored attribute in the pharmacy choice literature. Participants expressed their gratitude to pharmacists that exceeded their personal expectations of the pharmacist's professional role; which is also described as the 'helping professional' in the literature. ${ }^{[38]}$ Convenience 
was also important when choosing a pharmacy for some participants. However, an unexpected finding was highlighted by those describing the inconvenience of changing pharmacies, even if they were not entirely happy with the care received.

A strength of this study was its large participant size with varying chronic conditions and cultural backgrounds. Further analysis to identify the relationships across the three patron groups and participant demographics was not conducted and indeed would be challenging and inconclusive due to the inherent lack of generalisability in the approach. ${ }^{[39]}$ Whilst we relied on self-reported data, which can be influenced by participant bias, a standardised interview guide was used to ensure the key themes of interest were covered in a similar order. There is the risk that consumers were also users of hospital pharmacy services and talked about their experiences in this pharmacy sector. However, participants were specifically asked about their community pharmacy experience, and most people had at least one condition typically managed by medicines sourced from community pharmacies. To minimise the potential for interviewer bias, discussions were undertaken throughout the analysis between interviewers who had different research experience and professional backgrounds. This study recruited consumers with one or more chronic condition(s); therefore the findings may not translate to other pharmacy consumers, such as those with minor ailments. Further research to compare these two groups is warranted, as there are likely to be differences in the attributes of pharmacy choice. Finally, we did not explore the relative importance of these attributes, an area for future research.

The impact of convenience in influencing pharmacy choice is consistent with the literature. ${ }^{[12,22-24,40]}$ However, what this study has identified is that some people continued to use their regular pharmacy even after receiving what would be perceived as a lack of patientcentred care. This finding underscores the importance of subjective experiences in determining a person's choice. For example, the inconvenience of going elsewhere may over- 
ride the importance of patient-centred care. Furthermore, convenience may be more influential on pharmacy choice for consumers with chronic conditions and their carers due to the time or efforts needed to manage their conditions or roles; additional studies are needed to confirm this importance relative to patient-centred care for this population.

Alternatively, those consumers who patronise a conveniently located pharmacy with minimal patient-centred care may not be aware of better health services elsewhere. Indeed, Victoor et al. elaborated on this by suggesting that people may not have adequate information about different health providers to make a completely rational choice. ${ }^{[37]}$ It has also been suggested that if consumers have not experienced a higher level of service, such as extensive drug information consultation, then they are likely to remain satisfied with their current care. ${ }^{[23]}$ This study highlights that people with chronic conditions and their carers seemed to be unaware of the range of higher level community pharmacy services available to support them. ${ }^{[13,41]}$ Apart from dose administration aids and home medication deliveries, there was minimal discussion about choosing a pharmacy based on the provision of professional services. Therefore, more education about the standard of care and professional services that consumers should expect from their community pharmacy is needed; a recommendation identified previously in Australian practice. ${ }^{[25]}$

Pharmacists need to consider that if a professional service or patient-centred care is not provided, then the consumer may not know about it and therefore may not expect it. This in turn may influence an individual pharmacist's decision not to extend or improve their practice or service standards. Considering that some professional services are relatively new to Australian pharmacy practice, ${ }^{[8,9]}$ further targeted promotion to facilitate their uptake is essential. This requires a collaborative approach between professional bodies and community pharmacy staff. ${ }^{[42]}$ Given that a recent study has demonstrated that promotion of pharmacy 
services does not necessarily result in an uptake of use, ${ }^{[43]}$ this promotion should also aim to raise the profile of pharmacy as a healthcare destination. Based on the importance of patientcentred care for regular pharmacy users in this study, an emphasis on providing this approach to care is needed in community pharmacies. The public has questioned whether pharmacists are trained in patient-centred care. ${ }^{[44]}$ Pharmacy educators should consider this given we have identified patient-centred care as an important attribute. Community pharmacy staff should reflect on these findings, as patient-centred care can promote the formation of relationships, which in turn can facilitate regular patronage and increase professional satisfaction.

A variety of consumer personal traits such as cultural background and preferred spoken language could also influence a person's choice of pharmacy, suggesting that there is 'no such thing as the typical patient'. ${ }^{[37]}$ The importance of price is consistent with the literature, ${ }^{[15,22]}$ but is also subject to the individual consumer's circumstances, e.g. low income level or pensioner. Overall, our findings confirm that selecting a pharmacy is a subjective experience for each individual; hence community pharmacies need to meet the consumer's specific needs. The insights provided by participants can help inform community pharmacists as to what many people with chronic conditions and carers currently value from their pharmacy. People also need to be able to differentiate between the services that community pharmacies provide and match this to their priorities.

\section{CONCLUSION}

Patient-centred care, convenience, personal traits, price and specific needs can all influence a person's choice of pharmacy. As pricing and convenience in terms of location are more difficult to change, pharmacy staff can turn their attention to adopting a more patient-centred approach to care. This was an important attribute for participants with a chronic condition(s) when choosing a regular pharmacy. Consumers appreciated what they perceived to be quality 
service delivered by approachable pharmacy staff providing individualised medication supply and advice. Regular pharmacy patrons also valued the formation of relationships and a holistic approach to care.

These are positive findings for the community pharmacy sector. The profession is moving towards the implementation of more pharmacy services which can foster the development of, or enhance, relationships, an important influence on pharmacy patronage. As consumers with chronic conditions are often on multiple medications and require ongoing treatment, they are valuable consumers for pharmacy. It is therefore important that community pharmacy aims to meet their individual needs and provide the quality of care people want. 


\section{REFERENCES}

1. Blendon RJ et al. Common concerns amid diverse systems: health care experiences in five countries. Health Aff (Millwood), 2003; 22(3): 106-121.

2. Australian Commission on Safety and Quality in Health Care. Patient-centred care: Improving quality and safety through partnerships with patients and consumers. Sydney: ACSQHC; 2011.

3. Patient-centred care: Improving quality and safety by focusing care on patients and consumers — discussion paper. Canberra: Australian Commission on Safety and Quality on Health Care, 2010. http://www.safetyandquality.gov.au/our-work/patient-and-consumercentred-care/patient-and-consumer-centred-care-discussion-paper/ (Accessed 10 January 2013).

4. Crossing the Quality Chasm: A New Health System for the 21st Century. Washington, DC: National Academy Press, 2001. http://www.nap.edu/openbook.php?record_id=10027 (Accessed 10 January 2013). 5. George $\mathrm{P}$ et al. The evolving role of the community pharmacist in chronic disease management - a literature review. Ann Acad Med Singapore 2010; 39(11): 861-867.

6. Noncommunicable diseases country profiles 2011. Geneva: Switzerland: World Health Organization, 2011. http://www.who.int/nmh/publications/ncd_profiles2011/en/ (Accessed 10 January 2013).

7. Blalock SJ et al. The Effect of Community Pharmacy Based Interventions on Patient Health Outcomes: A Systematic Review. Med Care Res Rev 2013; 70(3): 235-66.

8. Program Specific Guidelines: Pharmacy Practice Incentives (PPI). Canberra: Australia, 2012. http://www.5cpa.com.au/iwovresources/documents/5CPA/Initiatives/PPI/Program_Specific_Guidelines/Pharmacy\%20Pract ice\%20Incentives\%20Program\%20Specific\%20Guildelines.pdf (Accessed 10 January 2013). 
9. Initatives and programs. The Pharmacy Guild of Australia, 2010.

http://www.5cpa.com.au/5CPA/Initiatives/Initiatives.page? (Accessed 10 January 2013).

10. White L, Klinner C. Service quality in community pharmacy: an exploration of determinants. Res Soc Admin Pharm 2012; 8(2): 122-132.

11. McMillan S.S et al. Patient centred approaches to health care: A systematic review of randomized controlled trials. Med Care Res Rev, 2013 (Accessed 1 August 2013).

12. Villako P, Raal A. A survey of Estonian consumer expectations from the pharmacy service and a comparison with the opinions of pharmacists. Pharm World Sci 2007; 29(5): 546-550.

13. Gastelurrutia MA et al. Customers' expectations and satisfaction with a pharmacy not providing advanced cognitive services. Pharm World Sci 2006; 28(6): 374-376.

14. Oparah AC, Kikanme LC. Consumer satisfaction with community pharmacies in Warri, Nigeria. Res Social Adm Pharm 2006; 2(4): 499-511.

15. Xu KT. Choice of and overall satisfaction with pharmacies among a communitydwelling elderly population. Med Care 2002; 40(12): 1283-1293.

16. Chen Y-C et al. Public views of community pharmacists in Taiwan. Int J Pharm Prac 2012; 20(3): 203-206.

17. Attree M. Patients' and relatives' experiences and perspectives of 'Good' and 'Not so Good' quality care. J Adv Nurs 2001; 33(4): 456-466.

18. Naik Panvelkar P et al. Measurement of patient satisfaction with community pharmacy services: a review. Pharm World Sci, 2009; 31(5): 525-537.

19. Um IS et al. Consumer perspectives about weight management services in a community pharmacy setting in NSW, Australia. Health Expect, 2012 (Accessed 10 Jan 2013) 
20. Teh R et al. Consumer perspectives of pharmacist-delivered healtn information and screening services. Int J Pharm Prac, 2001; 9(4): 261-267.

21. Taylor $\mathrm{J}$ et al. A community pharmacy-based cardiovascular screening service: views of service users and the public. Int J Pharm Prac, 2012; 20(5): 277-284.

22. Arneson DL et al. Patronage Motives of Community Pharmacy Patrons. Journal of Pharmaceutical Marketing \& Management, 1989; 4(2): 3-22.

23. Whitehead P et al. Patient drug information and consumer choice of pharmacy. Int $J$ Pharm Prac, 1999; 7(2): 71-79.

24. Phul S et al. Pharmacy services and patient choice: insights into differences between patient groups. Int J Pharm Prac, 2003; 11(4): 233-242.

25. Consumer experiences, needs and expectations of community pharmacy. The Pharmacy Guild of Australia, 2005. http://www.guild.org.au/sites/The_Guild/tabPharmacy_Services_and_Programs/Research_and_Development/Third\%20Agreement/2005501.page (Accessed 10 January 2013).

26. Lawrence Neuman W. Social Research Methods: Qualitative and Quantitative Apporaches. Boston USA: Pearson Education, Inc.; 2011.

27. Liamputtong P. Research methods in health: foundations for evidence-based practice. Sydney, NSW.: Oxford university press; 2010.

28. Sav A et al. Treatment burden among people with chronic illness: What are consumer health organisations saying? Chronic Illn, 2012 (Accessed 10 Jan 2013)

29. McMillan S et al. Community pharmacy in Australia: the health hub destination of the future. Res Soc Admin Pharm, 2012 (Accessed 10 Jan 2013)

30. Roulston K. Considering quality in qualitative interviewing. Qualitative Research, 2010; 10(2): 199-228. 
31. Boeije H. A Purposeful Approach to the Constant Comparative Method in the Analysis of Qualitative Interviews. Quality \& Quantity 2002; 36(4): 391-409.

32. Glaser BG. The Constant Comparative Method of Qualitative Analysis. Social Problems 1965; 12(4): 436-445.

33. Sim J, Wright C. Research in health care: concepts, designs and methods. United Kingdom Stanley Thornes (Publishers) Ltd; 2000.

34. Bowen GA. Naturalistic inquiry and the saturation concept: a research note. Qualitative Research 2008; 8(1): 137-152.

35. Morgan S, Yoder LH. A concept analysis of person-centered care. J Holist Nurs 2012; 30(1): 6-15.

36. Dancet EA et al. Patients from across Europe have similar views on patient-centred care: an international multilingual qualitative study in infertility care. Hum Reprod 2012; 27(6): 1702-1711.

37. Victoor A et al. Determinants of patient choice of healthcare providers: a scoping review. BMC Health Serv Res, 2012; 12(272).

38. Denhov A, Topor A. The components of helping relationships with professionals in psychiatry: users' perspective. Int J Soc Psychiatry 2012; 58(4): 417-424.

39. Lincoln Y.S, Guba E.G. Naturalistic Inquiry. USA: Sage Publications, Inc.; 1985.

40. Peterson GM et al. Public perceptions of the role of Australian pharmacists in cardiovascular disease. J Clin Pharm Ther, 2010; 35(6): 671-677.

41. Worley MM et al. Pharmacists' and patients' roles in the pharmacist-patient relationship: are pharmacists and patients reading from the same relationship script? Res Soc Admin Pharm, 2007; 3(1): 47-69. 
42. McMillan S.S. et al. Consumer and carer views of Australian community pharmacy practice: awareness, experiences and expectations. J Pharm Health Serv Res, 2013 (under review, submitted 5 March 2013)

43. Gidman $\mathrm{W}$ et al. Understanding public trust in services provided by community pharmacists relative to those provided by general practitioners: a qualitative study. $B M J$ Open, 2012; 2 (Accessed 10 Jan 2013, 2).

44. Rapport F et al. Through the Looking Glass: Public and Professional Perspectives on Patient-centred Professionalism in Modern-day Community Pharmacy. Forum: Qualitative Social Research, 2009; 11. 


\section{Panel 1: Interview questions related to pharmacy choice}

Why do you mainly go into the pharmacy? (e.g. purpose)

When you go to a pharmacy do you feel as though you are going there as a patient to get a health service or as a consumer to purchase a product?

Can you describe a good and a bad pharmacy experience?

Do you have a regular pharmacy? 
Table 1: Participant Data

\begin{tabular}{llcc}
\hline Participant characteristics & & $($ n) & $(\%)$ \\
\hline Carer/consumer & Consumer (C) & 69 & 71.1 \\
& Carer (CA) & 12 & 12.4 \\
& Carer/consumer (CC) & 16 & 16.5 \\
Race/cultural background & Aboriginal and Torres Strait Islander (IND) & 23 & 23.7 \\
& Culturally and linguistically diverse & 19 & 19.6 \\
& (CALD), e.g. Asian, Middle Eastern, & & \\
& European and Pacific Islander. & & \\
Interview method & Caucasian & 55 & 56.7 \\
& Face-to-Face & 49 & 50.5 \\
Location & Telephone & 48 & 49.5 \\
& Logan/ Beaudesert & 41 & 42.3 \\
& Mt Isa/ North West & 15 & 15.5 \\
Chronic condition(s)* & Northern Rivers & 21 & 21.6 \\
& Greater Perth & 20 & 20.6 \\
& One & 10 & 11.8 \\
& Two & 19 & 22.3 \\
& Three or more & 56 & 65.9 \\
\hline
\end{tabular}

*Data from consumer (C) and carer/consumer (CC) only 